\title{
Small-molecule-mediated reprogramming: a silver lining for regenerative medicine
}

\author{
Yohan Kim ${ }^{1,2}$, Jaemin Jeong ${ }^{1,2}$ and Dongho Choi ${ }^{1,2}$
}

\begin{abstract}
Techniques for reprogramming somatic cells create new opportunities for drug screening, disease modeling, artificial organ development, and cell therapy. The development of reprogramming techniques has grown exponentially since the discovery of induced pluripotent stem cells (iPSCs) by the transduction of four factors (OCT3/4, SOX2, C-MYC, and KLF4) in mouse embryonic fibroblasts. Initial studies on iPSCs led to direct-conversion techniques using transcription factors expressed mainly in target cells. However, reprogramming transcription factors with a virus risks integrating viral DNA and can be complicated by oncogenes. To address these problems, many researchers are developing reprogramming methods that use clinically applicable small molecules and growth factors. This review summarizes research trends in reprogramming cells using small molecules and growth factors, including their modes of action.
\end{abstract}

\section{Introduction}

Although modern medicine offers treatment methods for many diseases, such as Alzheimer's disease, heart attacks, and severe liver pathologies, many diseases remain difficult to treat. The most promising mordern technique is stem-cell therapy.

In 2006, Yamanaka reprogrammed somatic cells to become induced pluripotent stem cells (iPSCs) using four transcription factors, OCT4, SOX2, KLF4, and $c-M Y C^{1}$. Human iPSCs were developed using a similar induction strategy $^{2,3}$. Since their induction, iPSCs have been differentiated into neurons ${ }^{4-7}$, pancreatic cells ${ }^{8,9}$, osteogenic cells $^{9,10}$, hematopoietic cells ${ }^{10,11}$, cardiac cells ${ }^{12,13}$, adipocytes $^{14}$, vascular cells ${ }^{15}$, endothelial cells ${ }^{11}$, and hepatocytes $^{16,17}$. Direct conversion, which can transform one cell lineage into another while bypassing the pluripotent stage $^{18}$, became an alternative method for reprogramming cells into neuronal cells ${ }^{19}$ and cardiac cells ${ }^{20}$ in 2010 and into hepatocytes ${ }^{21}$ in 2011 (Fig. 1).

Correspondence: Dongho Choi (crane87@hanyang.ac.kr)

'Department of Surgery, Hanyang University College of Medicine, Seoul 04763, Korea

${ }^{2} \mathrm{HY}$ Indang Center of Regenerative Medicine and Stem Cell Research, Hanyang University, Seoul 04763, Korea

These authors contributed equally: Yohan Kim, Jaemin Jeong
Despite the development of efficient reprogramming, most methods are inappropriate for clinical applications because they carry the risk of integrating exogenous genetic factors or use oncogenes. Alternative approaches, such as those based on miRNA, nonviral genes, nonintegrative vectors, and small molecules, have been studied as possible solutions to the problems ${ }^{22}$.

Among these alternatives, small molecules are attractive options for clinical applications. Reprogramming using small molecules is inexpensive and easy to control in a concentration- and time-dependent manner. It offers a high level of cell permeability, ease of synthesis and standardization, and it is appropriate for mass-producing cells $^{23}$. This review summarizes research efforts involving small-molecule-mediated reprogramming of cells (SmCells) with growth factors from several organs.

\section{Induced pluripotent stem cells}

The efficiency of the first successful efforts to generate iPSCs with four factors was low. Hou et al. conducted studies to improve the efficiency of this process by adding small molecules ${ }^{24}$. Using various small-moleculescreening techniques, they confirmed that the H3K4 demethylation inhibitor tranylcypromine increases the generation efficiency of iPSCs and reported that 


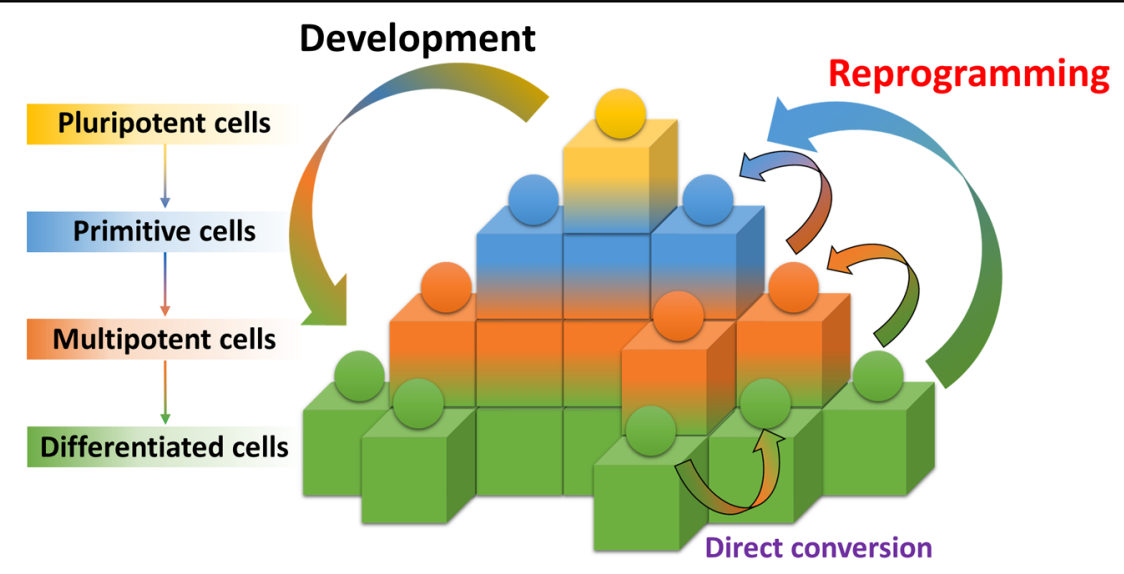

Fig. 1 Epigenetic landscape of cellular development and reprogramming. This staircase illustration depicts the fate of cells. The development process begins with pluripotency (yellow) and proceeds through the raw (blue) phase to the multifunctional (orange) and differentiated (green) phases. However, reprogramming can be induced from the differentiated stage to the primitive multipotency stage in response to extrinsic signaling, such as Yamanaka factors. Direct conversion of different cell types at various differentiation stages can be induced by forcing the overexpression of the main transcription factors of the targeted differentiation stage.

small-molecule-mediated iPSCs (SmiPSCs) could be generated using only Oct4, valproic acid (VPA), CHIR99021, 616452, and tranylcypromine (VC6T). Since then, this group has screened more than 10,000 small molecules to find a replacement for Oct4 and have found that forskolin (FSK), 2-methyl-5-hydroxytryptamine, and D4476 can replace Oct $4^{24}$. They also confirmed that green fluorescent protein (GFP)-positive clusters can be induced using only VC6T and FSK (VC6TF) in an Oct4-GFP reporter system. Adding 3-dezanafluronocin A (DZNep), an S-adenosylhomocysteine hydrolase inhibitor, increased the number of GFP-positive cells, demonstrating that FSK can serve as a substitute for Oct4 and that DZNep enhances the generation efficiency of iPSCs.

Several years later, the same team, Hou et al., addressed the problem posed by a heterogeneous population of fibroblasts that were reprogrammed at relatively low efficiency, and the possibility that a fibroblast subpopulation remains after reprogramming with small molecules $^{25}$. Whether reprogramming through small molecules can be performed in cells derived from ectoderm and endoderm lines was unknown. Therefore, Hou et al. conducted studies of small-molecule-based SmiPSC reprogramming from neural stem cells (NSCs) and small intestinal epithelial cells (IECs), which are ectoderm and endoderm cell types, respectively. First, they confirmed that they could reprogram SmiPSCs using small molecules in nonfibroblasts, and they devised a tracking system with fibroblast-specific protein 1 . After confirming the ability to reprogram other cell types, they attempted to reprogram NSCs (ectodermal lineage) with an Oct4-GFP reporter system. Attempts to reprogram NSCs into SmiPSCs using the previous VC6TF method had previously failed. However, Hou et al. found that epithelial clusters formed when EPO004777, the inhibitor of the H3K79 histone methyltransferase DOT1L, and Ch55, a retinoid acid receptor (RAR) agonist, were added and the concentration of 616452 was reduced. After adding DZNep to cells cultured for 20 days and switching to a $2 \mathrm{i}$ medium consisting of inhibitors of mitogen-activated protein kinase signaling and glycogen synthesis kinase-3 (GSK-3) used in ESC cultures ${ }^{26}$, compact and epithelioid colonies were detected, and the Oct4-GFP reporter gradually switched on and caused the formation of SmiPSC colonies. Next, to determine whether small-molecule reprogramming is applicable to endoderm cells, they reprogrammed IECs derived from Oct4-GFP transgenic mouse embryos by adding the RAR agonist AM580 to the previously used treatment cocktail followed by VC6TF and DZNep addition after 16 days. Epithelioid clusters were observed between 4 and 8 days after RAR treatment, and colony formation was evident after 15 days. After the conversion to $2 \mathrm{i}$ medium on day 40, Oct4-GFP-positive ESC-like colonies formed. The team then confirmed that the SmiPSCs generated by combining small molecules had the same characteristics as conventional iPSCs.

For both research projects, the scientific team used GSK-3, TGF- $\beta$, histone deacetylase, and monoamine oxidase inhibitors. The successful use of these factors suggests that activation of the Wnt signaling pathway, inhibition of TGF- $\beta$ signaling, and release of histones are important in the induction of SmiPSCs. Taken together, the data show that small-molecule-mediated reprogramming of all germline lineages into SmiPSCs appears to be possible, suggesting a new way of understanding pluripotency. 


\section{Brain cells}

There is currently no effective way to improve a patient's recovery from nervous system damage. However, recent studies suggest that adult neural stem/progenitor cells can regenerate and function against nervous system damage or disease ${ }^{27}$.

\section{Neural progenitor cells/neural stem cells}

In 2014, Cheng et al. reported that small-moleculemediated neural progenitor cells (SmNPCs) can be produced from mouse and human fibroblasts using a smallmolecule cocktail under hypoxic conditions ${ }^{28}$. They hypothesized that small molecules can induce the endogenous expression of $\operatorname{Sox} 2^{29,30}$ and that the hypoxic state could promote cellular reprogramming ${ }^{31}$. They found that reprogramming somatic cells into SmNPCs from both mice and humans was possible in a hypoxic state of $5 \% \mathrm{O}_{2}$, with VPA, CHIR99021, and Repsox functioning as the histone deacetylation inhibitor, glycogen synthase kinase, and TGF- $\beta$ pathway, respectively. The reprogrammed cells expressed NPC-specific markers and were able to differentiate into astrocytes, neurons, and oligodendrocytes.

A few years later, Zhang et al. reported reprogramming mouse fibroblasts into small-molecule-mediated neural stem cells (SmNSCs) using novel small-molecule combinations ${ }^{32}$. They proposed that a combination of small molecules involved in signaling nerve development and targeting epigenetic modifications could induce SmNSCs from somatic cells. They screened small-molecule combinations based on CHIR99021 and fibroblast growth factor (bFGF), which are known to enhance neural development $^{33,34}$, and LDN193189 and A83-01, which are known to inhibit mesoderm and endoderm development $^{5,35}$. In the first screening, they found that Hh-Ag 1.5 (which is a potent small-molecule agonist) and retinoic acid (RA) induced the coexpression of Nestin and Sox 2 in the cells at levels of $3.68 \%$ and $1.26 \%$, respectively. In follow-up screening studies, they confirmed that RG105 (a DNA methyltransferase inhibitor), parnate (a histone demethylase inhibitor), and SMER28 (an autophagy modulator) increased the number of generated coexpressing cells. Overall, the efficiency of generating coexpressing cells increased by $24.20-30.04 \%$ using a combination of 9 small molecules. The SmNSCs produced by this combination were able to proliferate for an extended period, differentiating into astrocytes, neurons, and oligodendrocytes.

\section{Neuronal cells/neurons}

Studies on small-molecule-mediated neuronal cells (SmNs) without a progenitor stage were carried out by $\mathrm{Hu}$ et al. in $2015^{36}$ (comprising the same scientists as the Cheng et al. team ${ }^{28}$ ), who conducted a screening test based on the results of their previous paper. Initially, human fibroblasts were treated with a combination of VPA, CHIR99021, and Repsox (VCR), which promoted neural differentiation of NPCs. The team did not observe neuron-like cells when they used only VCR. They therefore screened small molecules related to neuronal differentiation and found that a combination of FSK and VCR (VCRF) induced neuron-like cell morphology and the expression of b3-tubulin (TUJ1, a neuronal marker) on day 7. However, the morphology of the cells reprogrammed by VCRF differed from that of typical neurons; round and prominent cell bodies indicated that the reprogramming had been inefficient and partial. To improve the $\mathrm{SmN}$ reprogramming strategy, they used additional small molecules and found that a combination of SP600125 (a JNK inhibitor, S), Go 6983 (a PKC inhibitor, G), Y-27632 (a ROCK inhibitor, Y), and VCRF (VCRFSGY) produced TUJ1-positive cells with a neuronal morphology. The cells reprogrammed with VCRFSGY also expressed neuronal markers such as TUJ1, DCX, NEUN, and MAP2 after 7 days. Most of the reprogrammed cells survived for 10-12 days, but neuronal maturation ceased. Subsequently, $\mathrm{Hu}$ et al. found that the survival and maturation of reprogrammed cells increased significantly when they added dorsomorphin (an AMPK inhibitor, D) to CHIR99021 and FSK (CFD). More than $80 \%$ of the TUJ1-positive cells in these populations expressed vGLUT1, whereas GABAergic, cholinergic, and dopaminergic neurons were rarely found. The electrophysiological properties and gene expression patterns of these cells were similar to those of human iPSC-derived neurons and transcription factor-induced neurons. To determine whether this chemical induction method could be applied to neurological diseases, $\mathrm{Hu}$ et al. tested human dermal fibroblasts derived from patients with Alzheimer's disease. The induced cells derived from the Alzheimer's patients showed neuronal characteristics similar to those of normal reprogrammed cells. This result offers a new strategy for generating patient-specific neuronal cells and modeling neurological diseases.

At approximately the same time, Zhang et al. reprogrammed human astrocytes into functional SmNs using a small-molecule combination and sequential administration $^{37}$. They had previously reported that glial cells can directly differentiate into neurons through ectopic expression of NeuroD $1^{38}$. They conducted screening studies with 20 candidates that inhibit glial signaling pathways or activate neuronal signaling pathways to find small-molecule combinations that could replace these transcription factors. To increase reprogramming efficiency, they included several small molecules that modify DNA or histone structure. The results from the screening studies conducted with more than 100 conditions at different times and concentrations revealed that a cocktail of nine small molecules could reprogram human astrocytes 
into SmNs. First, human astrocytes were treated with LDN193189 $(0.25 \mu \mathrm{M})$, SB431542 $(5 \mu \mathrm{M}$, a selective ALK5 inhibitor), TTNPB $(0.5 \mu \mathrm{M}$, a RAR agonist), and thiazovivin $(0.5 \mu \mathrm{M}$, a ROCK inhibitor, Tzv) for 2 days. Zhang et al. used LDN193189, SB431542, and TTNPB to inhibit glial signaling pathways and activate neural signaling pathways $^{39-41}$ and Tzv to improve reprogramming efficiency and cell survival ${ }^{42,43}$. This initial small-molecule combination was replaced with CHIR99021 $(1.5 \mu \mathrm{M})$, DAPT $(5 \mu \mathrm{M}, \gamma$-secretase inhibitor), and VPA $(0.5 \mathrm{mM})$ 2 days after the initial phase. CHIR99021 and DAPT were used for $\mathrm{SmN}$ induction for 4 days ${ }^{44-46}$, and VPA was added to improve the reprogramming efficiency and remained in culture for only 2 days without inducing cell death $^{29}$. For the following 2 days, the team treated the cells with the smoothened agonist $(0.1 \mu \mathrm{M})$ and purmorphamine $(0.1 \mu \mathrm{M})$ to activate the sonic Hedgehog signaling pathway, which is the main factor in neural patterning ${ }^{47}$. On day 9 , they added the neurotrophic factor neurotrophin 3 and insulin-like growth factor 1 to enhance neuronal maturation. The resulting reprogrammed cells expressed the neuronal markers doublecortin, TUJ1, MAP2, and NEUN and survived for 4-5 months. This reprogramming scheme was not successful for use in spinal astrocytes; it had positive results only in cells originating in the brain. The reprogrammed cells had activity potentials and synchronous burst activity. The researchers then carried out lateral ventricle injections in mice to determine whether the cells could survive in vivo, and they reported that the reprogrammed cells survived in clusters in the brain 1 month after injection. However, the cells failed to undergo reprogramming directly in vivo. These studies show that smallmolecule cocktails promote cell plasticity and offer potential for use in transplantation.

Small molecules used in all the papers describing the reprogramming of SmPSCs, SmNSCs, and SmNs activate the Wnt signaling pathway and TGF- $\beta$ and BMP signaling inhibitors. Wnt signaling is known to stimulate neurogenesis $^{48}$, and inhibition of TGF- $\beta$ and BMP signaling reportedly induces differentiation of pluripotent cells ${ }^{30}$.

\section{Cardiac cells}

Recently, the generation of cardiomyocytes by directconversion techniques, such as the introduction of cardiac transcription factors (including Gata4, Mef2c, Tbx5, and $\operatorname{Hand}^{21,49,50}$ ) or a microRNA combination ${ }^{51}$, has been reported. The teams reported that the overexpression of Yamanaka factor causes heart development in the presence of a JAK inhibitor ${ }^{52}$. A few years later, the same team found that direct conversion into cardiomyocytelike cells was possible using a combination of Oct and small molecules ${ }^{53}$.
Based on those findings and those reported by $\mathrm{Hou}^{24}, \mathrm{Fu}$ et al. ${ }^{54}$ produced the first SmiPSCs using CHIR99021, Repsox, FSK, VPA, Parnate, and TTNPB (termed CRFVPT). Cell clusters similar to cardiomyocytes were developed during SmiPSC reprogramming and beating cells were unintentionally found between 6 and 8 days after treatment with CRFVPT. However, the beating cells were not observed after $\sim 1$ week in the SmiPSC-induction condition. Fu et al. therefore used a two-step strategy to promote the stable and effective induction of smallmolecule-mediated cardiomyocytes (SmCs), producing a cardiac-reprogramming medium based on the use of CRFVPT at the primary stage. First, they found that bFGF is not required for the generation of the SmCs. They also found that $15 \%$ fetal bovine serum (FBS) and 5\% knockout serum replacement (KSR) more efficiently generated beating cells than the combination of 10\% FBS and 10\% KSR that had been used to generate SmiPSCs. Moreover, they added N2 and B27 to increase the induction efficiency. Based on reports that matrix microstructures play important roles in cardiac reprogramming ${ }^{55}$, the scientists conducted the reprogramming in Matrigel-coated dishes, which allowed them to observe more beating cells. Because maintaining a cardiac-reprogramming medium for more than 16 days did not improve the efficiency, they removed the CRFVPT and added CHIR99021, PD0325901 (MEK1/2 inhibitor), LIF, and insulin, which are known maintenance factors for cardiomyocytes ${ }^{56-58}$. As a consequence, they found a significant increase in the number of beating cells. Then, $\mathrm{Fu}$ et al. identified the most important factors by removing one compound at a time from the CRFVPT combination, reporting that $\mathrm{C}, \mathrm{R}, \mathrm{F}$, and $\mathrm{V}$ play important roles in the induction of beating cells. They attempted SmC reprogramming of neonatal mouse tail-tip fibroblasts but found that the reprogramming efficiency was lower than it had been for the MEFs. Therefore, they added rolipram (a selective phosphodiesterase- 4 inhibitor) to the culture in the primary stage, which increased the reprogramming efficiency. These SmCs expressed cardiomyocyte markers such as $\alpha-$ actinin, cardiac troponin-T (cTnT), cardiac troponin-I, and $\alpha$-Major Histocompatibility Complex $(\alpha-\mathrm{MHC})$ and accurately exhibited cardiac electrophysiological characteristics. Next, the team confirmed that these cells expressed cardiac precursor markers at an early programming stage and could differentiate into smooth muscle cells and endothelial cells. The results suggest that this reprogramming method was successful because of a cardiac precursor stage similar to one observed during the natural development of myocardial cells.

In the same year, Cao et al. reported reprogramming human fibroblasts into SmCs using nine small-molecule combinations $^{59}$. To facilitate the tracking of $\mathrm{SmC}$ 
reprogramming, they labeled alpha myosin heavy chainGFP reporters in human foreskin fibroblasts. Guided by the cell activation and signaling-directed conversion paradigm $^{52,53}$, the Cao team used small molecules to induce or enhance cell reprogramming into cardiac cells. First, the researchers conducted screening studies on 89 small molecules known to promote reprogramming. They tested all the combinations against a small-molecule baseline cocktail of SB431542, CHIR99021, Parnate, and FSK, which are known to play important roles in direct conversion of cardiac cells ${ }^{53}$. The cells were treated with various small-molecule combinations for 6 days, after which the treatment was changed to an optimized cardiac-induction medium of activin $\mathrm{A}$, bone morphogenetic protein 4, vascular endothelial growth factor, and CHIR99021 for 5 days. Through these screening studies, they found a cocktail of 15 compounds $(15 \mathrm{C})$ that produced GFP-positive beating clusters. By removing the components of $15 \mathrm{C}$ one by one, they found that CHIR99021, A83-01, BIX01294 (histone methyltransferase inhibitor), AS8351 (epigenetic modulator), SC1 (extracellular signal-regulated kinase 1 and Ras GTPase inhibitor), Y27362 (ROCK inhibitor), and OAC2 (Oct4 activator) were the most important factors. They established that the most efficient combination for reprogramming skin cells into SmCs consisted of seven small molecules and two inhibitors, SU16 and JNJ-10198409. After 30 days of treatment with this small-molecule combination (9C), the cardiomyocyte marker cTnT was observed in $\sim 6.6 \%$ of the cells. The $9 \mathrm{C}$ cocktail also reprogrammed human fetal lung fibroblasts into SmCs. Through a series of processes, the mesoderm, cardiac progenitor cells, and cardiomyocytes are generated during cardiogenesis $^{60}$. This development was also observed in cells treated with $9 \mathrm{C}$. After treatment with cardiomyocyte induction medium, $\sim 27.9 \%$ of the cells expressed KDR, which is an important mesoderm marker. These cells expressed mesoderm, cardiac progenitor cells, and cardiomyocyte genes sequentially over time. Directly converting human cardiomyocyte-like cells using viruses has an extremely low reprogramming efficiency of $\sim 0.1 \%$, and the resulting cells are heterogeneous ${ }^{61}$. However, the production of SmCs produced via 9 C was $\sim 97 \%$ efficient, and the homogeneity was confirmed by single-cell quantitative reverse-transcription polymerase chain reaction (qRT-PCR) analysis. Approximately 45-50 days after reprogramming, intercellular electrical recordings showed strong action potential. In addition, most SmCs responded to caffeine, isoproterenol, and carbachol with a similar ventricular activity potential. These reprogrammed cells are therefore functional and have electrophysiological characteristics. The results show that more functional cells can be established using $9 \mathrm{C}$ than using previous methods.
SmC reprogramming inhibits Wnt signaling activation and TGFb in a manner similar to SmiPSC, SmNPC, SmNSs, and $\mathrm{SmN}$ reprogramming but is based on a wider variety of epigenetic modulators compared with those in the other cell types. According to $\mathrm{Fu}$ et al., SmC reprogramming is derived from the SmiPSC method; therefore, the signaling is most similar to that of SmiPSCs. Epigenetic modulation, cardiac development, and reprogramming are closely related ${ }^{62}$, suggesting that $\mathrm{SmCs}$ are induced through various epigenetic mechanisms similar to those that contribute to the production of SmiPSCs.

\section{Liver cells}

Many researchers are studying the possibility of using hepatic stem/progenitor cells for clinical applications ${ }^{63-65}$. Although the liver is a regenerating organ in vivo, no conclusive evidence of a liver progenitor has been reported. The theory of a liver progenitor assumes that regeneration occurs in the parenchyma and bile ducts ${ }^{66,67}$ or that mature hepatocytes are reprogrammed under chronic liver injury conditions ${ }^{68,69}$.

On the theoretical basis that reprogramming occurs in mature hepatocytes, Katsuda et al. conducted a reprogramming study to convert mature rat and mouse hepatocytes into small-molecule-mediated bipotent liver progenitor cells (SmLPs) in vitro ${ }^{70}$. They studied small molecules used in previous studies of iPSC generation $^{24,71,72}$ and found that PD0325901, Y-27632, A83-01, and CHIR99021 promoted mammary progenitor cells in culture. Therefore, to confirm the reprogramming of rat hepatocytes in vitro, they used these four small molecules in a small-hepatocyte culture medium, as reported in a previous paper ${ }^{73}$. In the presence of several combinations of the four small molecules, the cells proliferated, with the combination of Y-27632, A83-01, and CHIR99021 (YAC) showing the highest proliferative capacity. After 2 weeks, the number of mature rat hepatocytes treated with YAC increased $\sim 30$-fold, and the expression of proliferation markers and liver progenitor markers was upregulated. To identify the bipotential differentiation ability, an important feature of a liver progenitor, Katsuda et al. conducted hepatic differentiation experiments using a previously reported method $^{74}$. After hepatic differentiation, the cells showed a typical mature hepatocyte form, with canaliculi structures and increased expression of mature hepatocyte markers. The team also observed functions such as albumin secretion and Cyp1a activity. They conducted a separate differentiation study for the biliary epithelial cells, which are another potential liver progenitor. These differentiated cells expressed biliary epithelial cell markers and exhibited functional characteristics and a tubular form. These cells were stably reprogrammed in six donors and cultured without losing hepatic differentiation capacity after passage 20 . When the reprogrammed cells were 
A
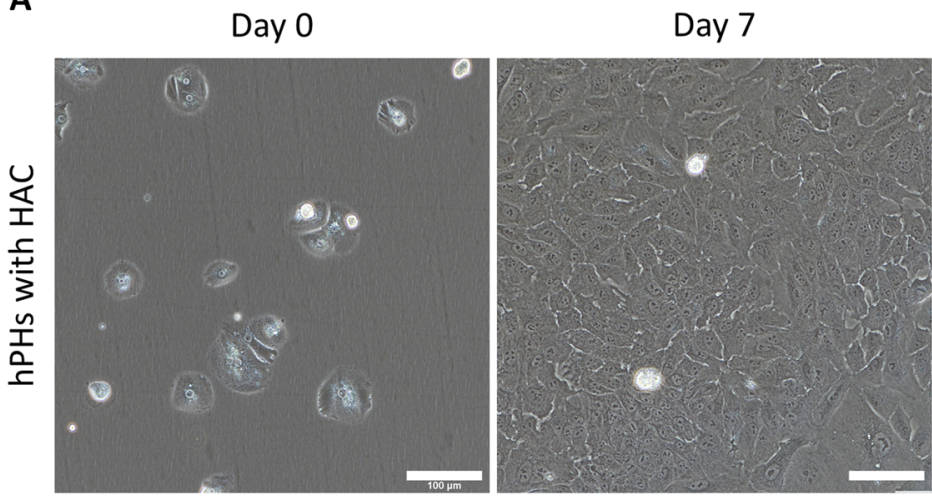

B

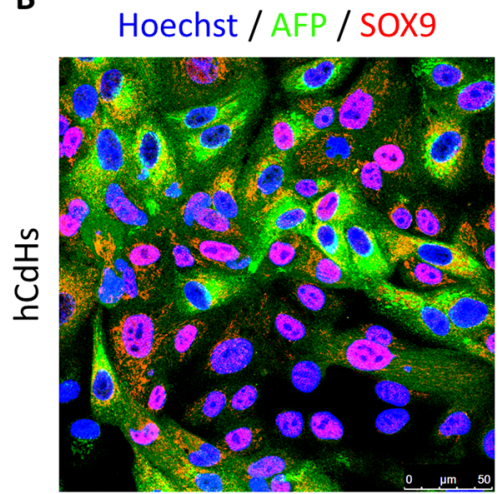

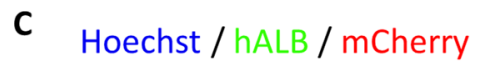

Hoechst / CK7 / mCherry
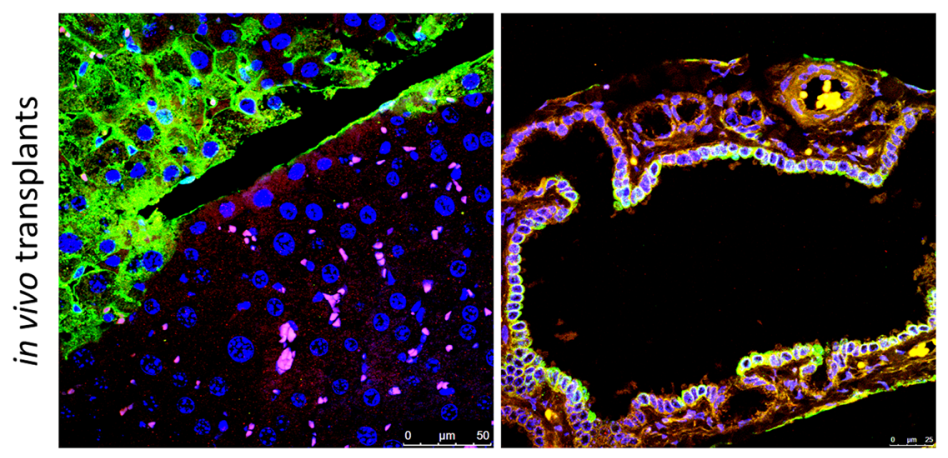

Fig. $\mathbf{2}$ hCdH generation and in vivo transplantation of hCdHs into liver disease model mice. a Freshly isolated human primary hepatocytes were cultured for 7 days in HAC-containing reprogramming medium. The morphology of human primary hepatocytes (hPHs) changes rapidly in the reprogramming medium, and the capacity to proliferate is acquired. Scale bars, $100 \mu \mathrm{m}$. b After generation, human chemically derived hepatic progenitors ( $\mathrm{hCdHs}$ ) effectively express the progenitor markers alpha fetoprotein (AFP) and SOX9. Scale bars, 50 um. c A liver paraffin Section 3 weeks after mCherry-tagged hCdHs were transplanted into Alb-TRECK/SCID mice. Liver sections were stained with human albumin (ALB, green, left), human cytokeratin 7 (CK7, green, right), and mCherry (red, both), and the nuclei were counterstained with Hoechst. Scale bars, 50 $\mu$ m.

transplanted into uPA transgenic mice crossed with severe combined immunodeficiency (SCID) mice (cDNAuPA/SCID mice $)^{75}, 75-90 \%$ of the cells were repopulated without forming tumors. Although the reprogramming methods using YAC were efficient, they failed in humans.

Drawing on the results of these studies, our group reprogrammed small molecules to transform human adult hepatocytes into $\mathrm{SmLPs}^{76}$. Using the cocktail and reprogramming method of Katsuda et al. ${ }^{70}$, we further investigated the factors that increased conversion efficiency. We found that a combination of hepatocyte growth factor (HGF), which is important for hepatic progenitor regeneration and maintenance ${ }^{64,77}$, A83-01, and CHIR99021 (HAC), induced reprogramming of the human hepatocytes (Fig. 2a). These chemically derived human hepatic progenitors (hCdHs) showed rapid proliferative capacity and expressed hepatic progenitor markers 10-15 days after HAC treatment (Fig. 2b). The hCdHs showed a significant increase in stem-cell-related gene set expression and were stably reprogrammed in 6 donors with maintained chromosomal integrity. Identification of the downstream signaling messengers of the HGF receptor (the MET signaling pathway) confirmed the role of HGF in generating hCdHs. As a result, we observed that, among downstream targets of MET, ERK phosphorylation was increased in the presence of HGF during the reprogramming stage. According to the results from additional experiments, we confirmed that the hCdHs were generated through an ERK1/2 mechanism. To measure the bipotent differentiation capacity of these hCdHs, differentiation experiments were carried out following the protocol found in a previous paper ${ }^{74}$. After hepatic differentiation, the expression of mature hepatocyte-specific genes increased significantly, and we observed that these differentiated cells had the functional characteristics of mature hepatocytes in albumin secretions and during urea synthesis and CYP1A2 activity, and upon glycogen staining. When bile epithelial cell differentiation was induced via a 3D culture system to confirm the bipotent differentiation potential of the $\mathrm{hCdHs}^{78}$, the hCdHs formed a tubular structure, and the expression of a biliary marker was increased. These $\mathrm{hCdHs}$ also maintained 
proliferation capacity, expression of progenitor markers, and hepatic differentiation capacity for more than 10 passages. To further characterize the hepatic differentiation of these $\mathrm{hCdHs}$, we compared their global gene expression with that of other hepatocyte-like cells. The results from a clustering analysis revealed that, among hepatocyte-like cells, the hepatically differentiated hCdHs showed the highest correlation with mature hepatocytes, such as human iPSC-derived hepatocytes, ESC-derived hepatocytes, direct-converted hepatocytes, and liver progenitor-like cells. Next, we transplanted the hCdHs into Alb-TRECK/SCID mice and NOD CgPrkdc $^{\text {scid }}{ } / 2 \mathrm{rg}^{\mathrm{tm} 1 \mathrm{Wjl}} / \mathrm{SzJ}$ mice (Fig. 2c). The results show that the hCdHs repopulated as much as $20 \%$ of the diseased livers, and the levels of serum human albumin and alpha-1 antitrypsin were increased after transplantation. The hCdHs differentiated into hepatocytes and biliary epithelial cells in vivo. Taken together, our results show that our HAC reprogramming system for human hepatocytes has bipotent differentiation ability in vitro and in vivo.

A comparable study by $\mathrm{Fu}$ et al. revealed that the reprogramming of human hepatocytes was completed within a month ${ }^{79}$. This group reported reprogramming SmLPs via SIRT1 signaling. They removed $\mathrm{CD} 24^{+}$or $\mathrm{EpCAM}^{+}$cells from human hepatocytes using FACS sorting and cultured the sorted cells with transition and expansion medium (TEM) containing HGF, EGF, Y27632, CHIR99021, A83-01, sphingosine-1-phosphate (an activator of GPR, S1P), and lysophosphatidic acid (an activator of EDG, LPA). Under TEM culture conditions, human hepatocytes were rapidly reprogrammed, and the expression of progenitor markers with normal diploid karyotypes was increased. These SmLPs could be cultured for as many as 10 passages in seven of the nine patients and survived through passage 20 with proliferation capacity in three patients. The proliferation ability was significantly reduced in the hepatocytes cultured with nicotinamide, which is known to inhibit the sirtuin protein family, and the expression of SIRT1 was increased under TEM conditions. Thus, supported with the results from additional inhibitor experiments, $\mathrm{Fu}$ et al. found that SIRT1 is an important factor in human hepatocytes. After hepatic differentiation, the SmLPs differentiated into mature hepatocytes with a repopulation efficiency of $7-16 \%$ when they were transplanted into $\mathrm{Fah}^{-1-} \mathrm{Rah} 2^{-/-}$ $(\mathrm{F} / \mathrm{R})$ mice. Next, the team produced hepatitis B virus (HBV)-infected 3D spheroid models of the SmLPs in a 3D culture. Their HBV spheroids effectively expressed viral factors such as retinoid-X receptor (RXR) A, HNF4A, and the viral receptor NTCP, and they extensively characterized HBV. Additionally, this HBV model was confirmed to be reproducible in HBV patient-derived SmLPs. Ultimately, anti-HBV studies using a Cas9 system and this
HBV spheroid model were possible. Reprogrammed cells can therefore be suitable cell sources for disease modeling.

Similar approaches to reprogramming human hepatocytes into SmLPSs with proliferation capacity have also been reported by Zhang et al. ${ }^{80}$, who modified the culture medium on the assumption that the Wnt3a, Rspo1, noggin, and FSK included in human liver isolation medium (HLIM), which also contained FGF10, EGF, HGF, human gastrin I, A83-01, Y-27632, were not optimized for the culture of human hepatocytes. They found that the proliferation of human hepatocytes was significantly reduced after the Wnt3a-conditioned medium was removed from the HLIM. Conversely, when Rspo1, Noggin, and FSK were removed from the HLIM, the proliferation of the human hepatocytes increased significantly. This result confirmed that Wnt3a is critical for the proliferation of human hepatocytes and that Rspo1, Noggin, and FSK should be removed from HLIM to enable the proliferation of human hepatocytes. Approximately $77 \%$ of the human hepatocytes cultured with modified HLIM had KI67 + cells within 3 day and maintained the expression of progenitor markers for more than a month. These proliferating cells were able to undergo hepatocyte maturation under 3D culture conditions and showed a significant increase in the expression of hepatic markers such as ALB and AAT. This reprogramming was possible in all 7 donors, with younger donors showing higher proliferation abilities. The human hepatocytes cultured in modified HLIM and transplanted into FRG mice showed a high survival rate and repopulated approximately $64 \%$ of the surviving mice. In contrast to that of our findings ${ }^{76}$, the histology report by Zhang et al. showed that all these cells differentiated into hepatocytes only in vivo. The results of their study suggest a way to produce proliferating cells that can differentiate into mature hepatocytes through a medium containing the Wnt3a signaling pathway components.

Parallel finding of Zhang et al., we found that HGF, which regulates cell growth, cell motility, and morphogenesis $^{81}$, plays an essential role in the reprogramming of human hepatocytes, and Wnt- $\beta$-catenin, which is closely related to liver development and health ${ }^{82}$, also plays a major role in the reprogramming of SmLPs.

\section{Other cell types \\ Adipocytes}

Brown adipose tissue (BAT) has recently received attention as the source for a potential treatment for obesity and metabolic diseases ${ }^{83,84}$. Whereas white adipose tissue accumulates energy, BAT dissipates energy in the form of heat, which is related to the energy homeostasis of the body $^{85}$. In 2008, PRDM16 was reported to be a major factor in the conversion of skeletal myoblasts to 
small-molecule-mediated BAT-like adipocytes $(\mathrm{SmBAs})^{86}$. Based on that report, Nie et al. conducted a highthroughput phenotypic screening of 20,000 compounds in skeletal myoblasts to find small molecules that induce the overexpression of PRDM16 ${ }^{87}$. As a result, adipogenesis was observed in the cells exposed to some of these compounds, including bexarotene (a RAR agonist, Bex). Citing the report that RXR induced UCP1 in brown adipocytes ${ }^{8}$, the Nie team studied whether Bex/RXR causes brown adipogenesis. Their experiments confirmed that adipogenic reprogramming occurred two days after the cells were treated with Bex. The reprogrammed cells showed increased expression of Prdm16 and brown adipocyte markers such as UCP1 and suppressed white adipogenesis. Through further experiments, the group also found that $R x r \alpha / \gamma$ activation is essential for the induction of BAT. To confirm the effect of Bex in vivo, they administered Bex orally to mice fed a high-fat-diet for 4 weeks, which resulted in less weight gain compared with that of a control group. In conclusion, Nie et al. reported methods that were all based on the use of small molecules to induce SmBA production in vitro and in vivo.

\section{$\beta$ cells}

The induction of the differentiation of cells from the liver in the endocrine pancreas is an attractive strategy because the liver and pancreas have the same precursors at the embryonic endoderm stage ${ }^{89}$. Liu et al. therefore reprogrammed rat liver epithelial stem-cell-like WB-F344 cells (WB cells) into small-molecule-mediated insulinsecreting cells (SmISCs) in three stages ${ }^{90}$. First, they treated WB cells with 5-aza-2'-deoxycytidine (an inhibitor of DNA methylase, 5-AZA) for 2 days, followed by trichostatin A (a regulator of chromatin remodeling, TSA) for 1 day. In the first step, cell proliferation was reduced, the cells became spindle shaped, and expression of the liver marker ceased. In the second step, they treated the cells with $1 \times$ insulin transferrin, selenite, and retinoic acid (RA) for 7 days. At that stage, the size of the cell decreased, and the gene expression of Pdx1, a pancreatic progenitor, increased. Gene expression in stage 2 was similar to the expression observed during the early pancreatic stage during development. In the final stage, the cells were treated with nicotinamide for 7 days, which produced mature SmISCs. After maturation, the cells increased their expression of islet-specific markers, and insulin genes were also activated. When these SmISCs were transplanted into streptozotocin-treated mice, the mouse glucose levels were maintained, and the serum insulin concentration more than doubled compared with that of the control group. Liu et al. therefore successfully used small molecules to reprogram hepatic stem-like cells into pancreatic progenitors expressing $\mathrm{Pdx} 1$ and insulinproducing cells that functioned in vitro and in vivo.

\section{Small molecules}

As discussed above, various cell types can be reprogrammed using combinations of small molecules (Fig. 3 and Table 1). The molecular and biological mechanisms of reprogramming some types of cells into other types are largely understood, but reprogramming via small molecules provides further information about these mechanisms (Table 2).

As shown in the reports presented above, CHIR99021 is used in almost all small-molecule cell reprogramming. CHIR99021 activates the Wnt signaling pathway by

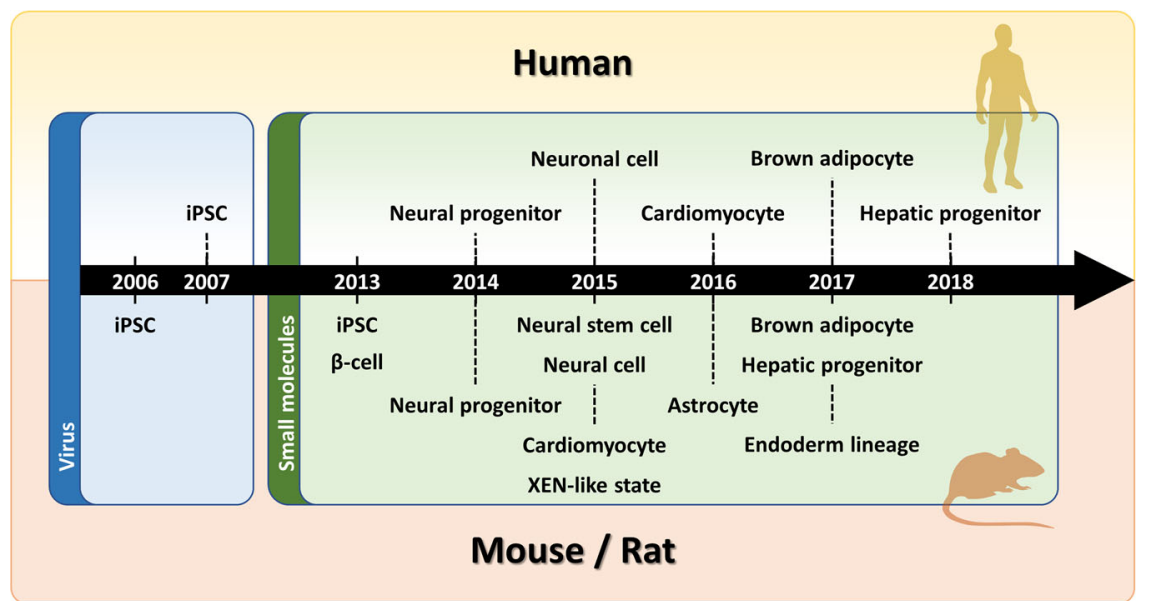

Fig. 3 Historical timeline of small-molecule-mediated reprogramming. Reprogramming efforts using small molecules by year. In 2006, iPSCs were created using viruses (blue), and since 2013, small-molecule reprogramming (green) has been developed. The timeline is divided into mouse/ rat and human phases. Immediately after the reprogramming of $\beta$ cells and iPSCs was announced in 2013, the reprogramming of other cells was reported. 
Table 1 Cellular reprogramming with small molecules.

\begin{tabular}{|c|c|c|c|c|}
\hline Cell type & Small molecule & Source & Species & Reference \\
\hline \multirow{2}{*}{$\begin{array}{l}\text { induced pluripotent } \\
\text { stem cells }\end{array}$} & VPA, CHIR99021, 616452, Tranylcypromine & Fibroblast & Mouse & 24 \\
\hline & $\begin{array}{l}\text { VPA, CHIR99021, 616452, Tranylcypromine, Forskolin, DZNep, } \\
\text { EPZ004777, Ch55, AM580 }\end{array}$ & $\begin{array}{l}\text { Fibroblast } \\
\text { Neural stem cells } \\
\text { Small intestinal } \\
\text { epithelial cells }\end{array}$ & Mouse & 25 \\
\hline \multirow[t]{2}{*}{ Neural stem cells } & $\begin{array}{l}\text { SB-431542, LDN193189, CHIR99021, PD0325901, Pifithrin-a, } \\
\text { Forskolin }\end{array}$ & Fibroblast & $\begin{array}{l}\text { Mouse } \\
\text { Human }\end{array}$ & 28 \\
\hline & $\begin{array}{l}\text { A83-01, LDN193189, CHIR99021, Hh-Ag 1.5, Retinoic acid, SMER28, } \\
\text { RG108, Parnate, bFGF }\end{array}$ & Fibroblast & Mouse & 32 \\
\hline \multirow[t]{2}{*}{ Neuronal cells } & $\begin{array}{l}\text { CHIR99021, Dorsomorphin, Forskolin, Go6983, RepSox, SP600125, } \\
\text { VPA, Y-27632 }\end{array}$ & Fibroblast & Human & 36 \\
\hline & $\begin{array}{l}\text { CHIR99021, DAPT, LDN193189, Purmorphamine, SAG, SB-431542, } \\
\text { TTNPB, TZV, VPA }\end{array}$ & Astrocyte & Human & 37 \\
\hline \multirow[t]{2}{*}{ Cardiomyocyte } & $\begin{array}{l}\text { CHIR99021, Forskolin, Insulin, LIF, Parnate, PD0325901, RepSox, } \\
\text { TTNBP, Vitamin C, VPA }\end{array}$ & Fibroblast & Mouse & 54 \\
\hline & $\begin{array}{l}\text { A83-01, AS8351, BIX01294, CHIR99021, JNJ-10198409, OAC2, SC1, } \\
\text { SU16F, Y-27632 }\end{array}$ & Fibroblast & Human & 59 \\
\hline \multirow[t]{4}{*}{ Hepatic progenitor } & Y-27632, A83-01, CHIR99021, EGF & Hepatocyte & Mouse & 70 \\
\hline & HGF, EGF, A83-01, CHIR99021 & Hepatocyte & Human & 76 \\
\hline & HGF, EGF, Y-27632, CHIR99021, A83-01, S1P, LPA & Hepatocyte & Human & 79 \\
\hline & FGF10, EGF, HGF, human gastrin I, A83-01, Y-27632, Wnt3a & Hepatocyte & Human & 80 \\
\hline Adipocyte & $\begin{array}{l}\text { Bexarotene, Dexamethasone, Indomethacin, Insulin, Iso- } \\
\text { buylmethylxanthine, T3 }\end{array}$ & Skeletal myoblast & Mouse & 87 \\
\hline$\beta$-cell & 5-AZA, TSA, Retionic acid, Nicotinamide & $\begin{array}{l}\text { Liver epithelial stem- } \\
\text { like cells }\end{array}$ & Rat & 90 \\
\hline
\end{tabular}

inhibiting GSK3 ${ }^{91}$ (Fig. 4). During Wnt signaling, GSK-3 binds to $\beta$-catenin and degrades it via phosphorylation, inhibiting transcription. CHIR99021 selectively inhibits GSK-3, allowing $\beta$-catenin to enter the nucleus and proceed with transcription. Because the target gene of this transcription consists of genes related to the cell cycle, CHIR99021 induces self-renewal of stem cells. This process suggests that the Wnt pathway plays an important role in the induction during the primitive stage.

TGF- $\beta$ inhibitors, such as 616452, A83-01, Repsox, and SB-431542, are used to reprogram almost all cells. The TGF- $\beta$ inhibitors used for small-molecule-mediated reprogramming follow mechanisms that inhibit TGF- $\beta$ receptors. TGF- $\beta$ phosphorylates SMAD2/3, which then binds to SMAD4 and enters the nucleus to regulate transcription. TGF- $\beta$ signaling plays an important role in regulating cell growth, differentiation and development, and TGF- $\beta$ inhibitors are expected to confer stem-cell properties by inhibiting these regulatory pathways. Additionally, inhibition of the TGF- $\beta$ pathway has been reported to replace Sox2 and induce Nanog during reprogramming ${ }^{30}$, and several stem-cell factors are known to play important roles in regeneration ${ }^{34,92}$.

RAR agonists, including AM580, Bex, Ch55, RA, and TTNPB, have also been used in the reprogramming of various cells. RAR signaling plays a critical role in molecular reprogramming and leads to pluripotency through synergistic interactions ${ }^{93}$. It is also known to induce differentiation of stem cells, leading them to various cell fates $^{94,95}$. Small molecules that regulate the methylation/ acetylation of DNA or histones, such as 5-AZA, AS8351, BIX01294, DZNep, RG108, TSA, and VPA, are also important to reprogramming cocktails. The induction of epigenetic changes opens chromatin, activates various pathways related to reprogramming, increases the population of Oct4-positive cells, and improves reprogramming efficiency ${ }^{29,96-98}$. It has also been demonstrated that frequently used ROCK inhibitors reduce senescence ${ }^{99}$.

Reprogramming of functional cells using these small molecules is more likely to be applied to future 
Table 2 Small molecules.

\begin{tabular}{|c|c|c|c|}
\hline Chemical component & Mechanism & Reprogrammed cells & References \\
\hline \multirow[t]{3}{*}{ 616452, RepSox } & \multirow[t]{3}{*}{ Inhibitor of TGF- $\beta$ RI } & iPSCs & 24,25 \\
\hline & & Neuronal cells & 36 \\
\hline & & Cardiomyocytes & 54 \\
\hline 5-Aza-2'-deoxycytidine & DNA methyltransferase activity inhibitor & $\beta$-cells & 90 \\
\hline \multirow[t]{3}{*}{ A83-01 } & \multirow[t]{3}{*}{ TGF- $\beta$ RI kinase inhibitor IV } & Neural stem cells & 32 \\
\hline & & Cardiomyocytes & 59 \\
\hline & & Hepatic progenitors & $70,76,79,80$ \\
\hline AM580 & RARa agonist & iPSCs & 25 \\
\hline AS8351 & histone demethylase (HDM) inhibitor & Cardiomyocytes & 59 \\
\hline Bexarotene & RAR agonist & Adipocytes & 87 \\
\hline bFGF & Basic fibroblast growth factor & Neural stem cells & 32 \\
\hline BIX01294 & Histone methyltransferase inhibitor & Cardiomyocytes & 59 \\
\hline Ch55 & RAR agonist & iPSCs & 25 \\
\hline \multirow[t]{5}{*}{ CHIR99021 } & \multirow[t]{5}{*}{ GSK-3 inhibitor } & iPSCs & 24,25 \\
\hline & & Neural stem cells & 28,32 \\
\hline & & Neuronal cells & 36,37 \\
\hline & & Cardiomyocytes & 54,59 \\
\hline & & Hepatic progenitors & $70,76,79$ \\
\hline DAPT & $\gamma$-secretase inhibitor & Neuronal cells & 37 \\
\hline Dorsomorphin & BMP inhibitor & Neuronal cells & 36 \\
\hline DZNep & Histone Methyltransferase EZH2 Inhibitor & iPSCs & 25 \\
\hline EGF & Epidermal growth factor & Hepatic progenitors & $70,76,79,80$ \\
\hline EPZ004807 & DOT1L inhibitor & iPSCs & 25 \\
\hline FGF10 & Fibroblast growth factor 10 & Hepatic progenitors & 80 \\
\hline \multirow[t]{4}{*}{ Forskolin } & \multirow[t]{4}{*}{ Adenylyl cyclase activator } & iPSCs & 25 \\
\hline & & Neural stem cells & 28 \\
\hline & & Neuronal cells & 36 \\
\hline & & Cardiomyocytes & 54 \\
\hline Go 6983 & PKC inhibitor & Neuronal cells & 36 \\
\hline HGF & Hepatocyte growth factor & Hepatic progenitors & $76,79,80$ \\
\hline Hh-Ag 1.5 & Hh Signaling Pathway Agonist & Neural stem cells & 32 \\
\hline human gastrin I & CCK2 receptor agonist & Hepatic progenitors & 80 \\
\hline Insulin & Endogenous peptide agonist & Cardiomyocytes & 54 \\
\hline JNJ-10198409 & PDGFR Tyrosine Kinase Inhibitor IV & Cardiomyocytes & 59 \\
\hline \multirow[t]{2}{*}{ LDN193189 } & \multirow[t]{2}{*}{ ALK2 and ALK3 inhibitor } & Neural stem cells & 28,32 \\
\hline & & Neuronal cells & 37 \\
\hline LIF & Leukemia Inhibitory Factor & Cardiomyocytes & 54 \\
\hline LPA & A ligand activator for EDG-2, EDG-4, and EDG-7 & Hepatic progenitors & 79 \\
\hline Nicotinamide & PARP-1 inhibitor & $\beta$-cells & 90 \\
\hline OAC2 & Oct4 activator & Cardiomyocytes & 59 \\
\hline \multirow[t]{3}{*}{ Parnate, Tranylcypromine } & \multirow[t]{3}{*}{ Monoamine oxidase inhibitor, LSD1 inhibitor } & iPSCs & 24,25 \\
\hline & & Neural stem cells & 32 \\
\hline & & Cardiomyocytes & 54 \\
\hline \multirow[t]{2}{*}{ PD0325901 } & \multirow[t]{2}{*}{ Potent inhibitor of MEK1/2 } & Neural stem cells & 28 \\
\hline & & Cardiomyocytes & 54 \\
\hline Pifithrin-a & p53 inhibitor & Neural stem cells & 28 \\
\hline Purmorphamine & Smo receptor agonist & Neuronal cells & 37 \\
\hline \multirow[t]{2}{*}{ Retinoic acid } & \multirow[t]{2}{*}{ Endogenous retinoic acid receptor agonist } & Neural stem cells & 32 \\
\hline & & $\beta$-cells & 90 \\
\hline RG108 & Non-nucleoside DNA methyltransferase inhibitor & Neural stem cells & 32 \\
\hline Sphingosine-1-Phosphate & A ligand for EDG-1 and EDG-3 and activator of GPR3, GPR6, and GPR12 & Hepatic progenitors & 79 \\
\hline SAG & Hedgehog signaling activator & Neuronal cells & 37 \\
\hline \multirow[t]{2}{*}{ SB-431542 } & \multirow[t]{2}{*}{ Inhibitor of TGF- $\beta$ RI, ALK4 and ALK7 } & Neural stem cells & 28 \\
\hline & & Neuronal cells & 37 \\
\hline
\end{tabular}


Table 2 continued

\begin{tabular}{|c|c|c|c|}
\hline Chemical component & Mechanism & Reprogrammed cells & References \\
\hline $\mathrm{SC} 1$ & Dual inhibition of extracellular signal-regulated kinase 1 and Ras GTPase & Cardiomyocytes & 59 \\
\hline SMER28 & Positive regulator of autophagy & Neural stem cells & 32 \\
\hline SP600125 & JNK inhibitor & Neuronal cells & 36 \\
\hline SU16F & PDGFRß inhibitor & Cardiomyocytes & 59 \\
\hline Trichostatin A & Histone deacetylase inhibitor & $\beta$-cells & 90 \\
\hline \multirow[t]{2}{*}{ TTNPB } & \multirow[t]{2}{*}{ RAR agonist } & Neuronal cells & 37 \\
\hline & & Cardiomyocytes & 54 \\
\hline Thiazovivin & ROCK inhibitor & Neuronal cells & 37 \\
\hline \multirow[t]{3}{*}{ Valproic acid } & \multirow[t]{3}{*}{ Histone deacetylase inhibitor } & iPSCS & 24,25 \\
\hline & & Neuronal cells & 36,37 \\
\hline & & Cardiomyocytes & 54 \\
\hline Wnt3a & Wnt family & Hepatic progenitors & 80 \\
\hline \multirow[t]{3}{*}{ Y-27632 } & \multirow[t]{3}{*}{ ROCK inhibitor } & Neuronal cells & 36 \\
\hline & & Cardiomyocytes & 59 \\
\hline & & Hepatic progenitors & $70,79,80$ \\
\hline
\end{tabular}

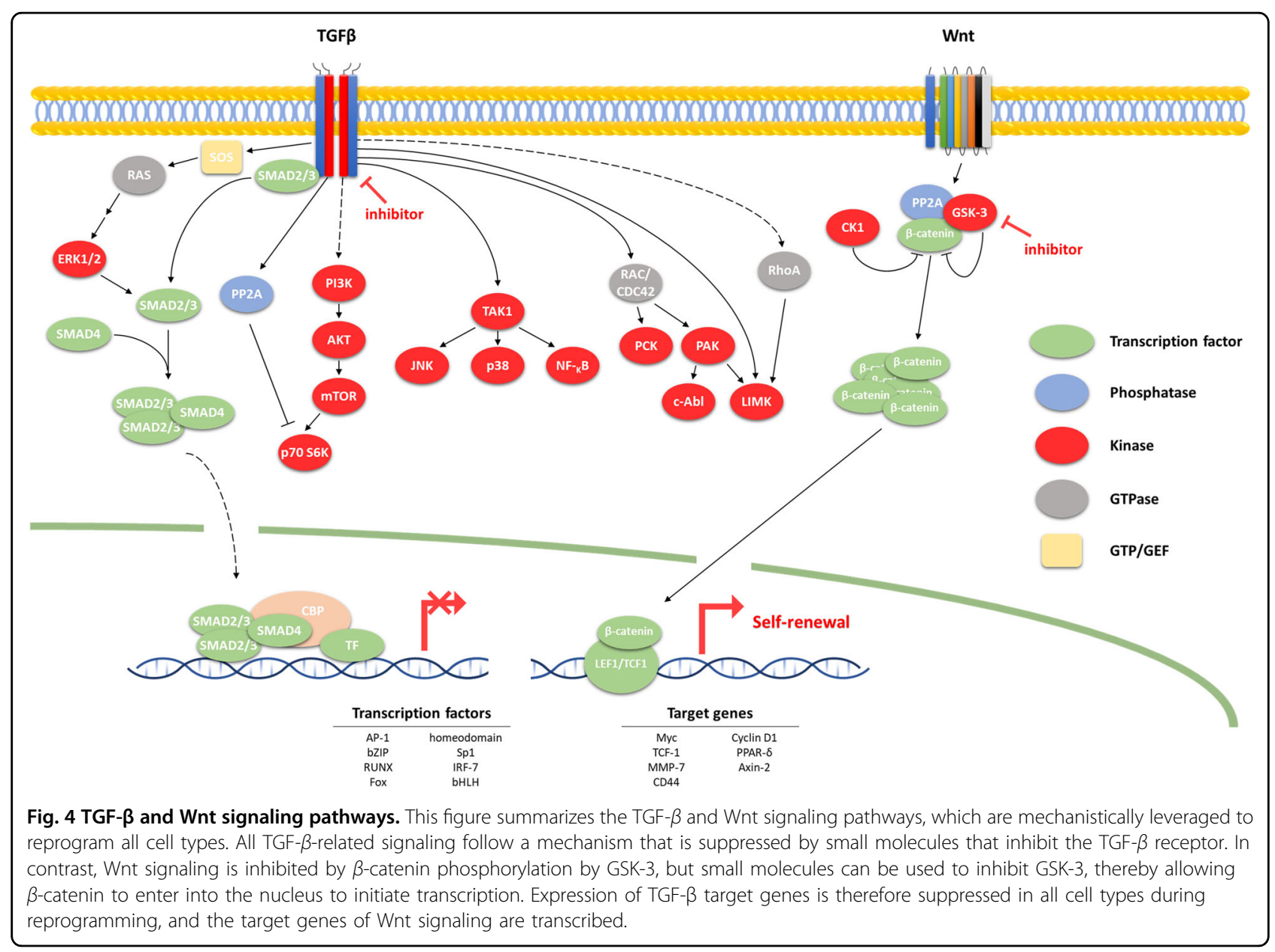

regenerative medicine than other stem-cell sources (Fig. 5). The risk of tumor formation can be reduced by avoiding the problem of genetic integration posed by viruses and using existing transcription factors. Furthermore, small molecules are relatively easy to study in reprogramming in vivo and are amenable to oral 


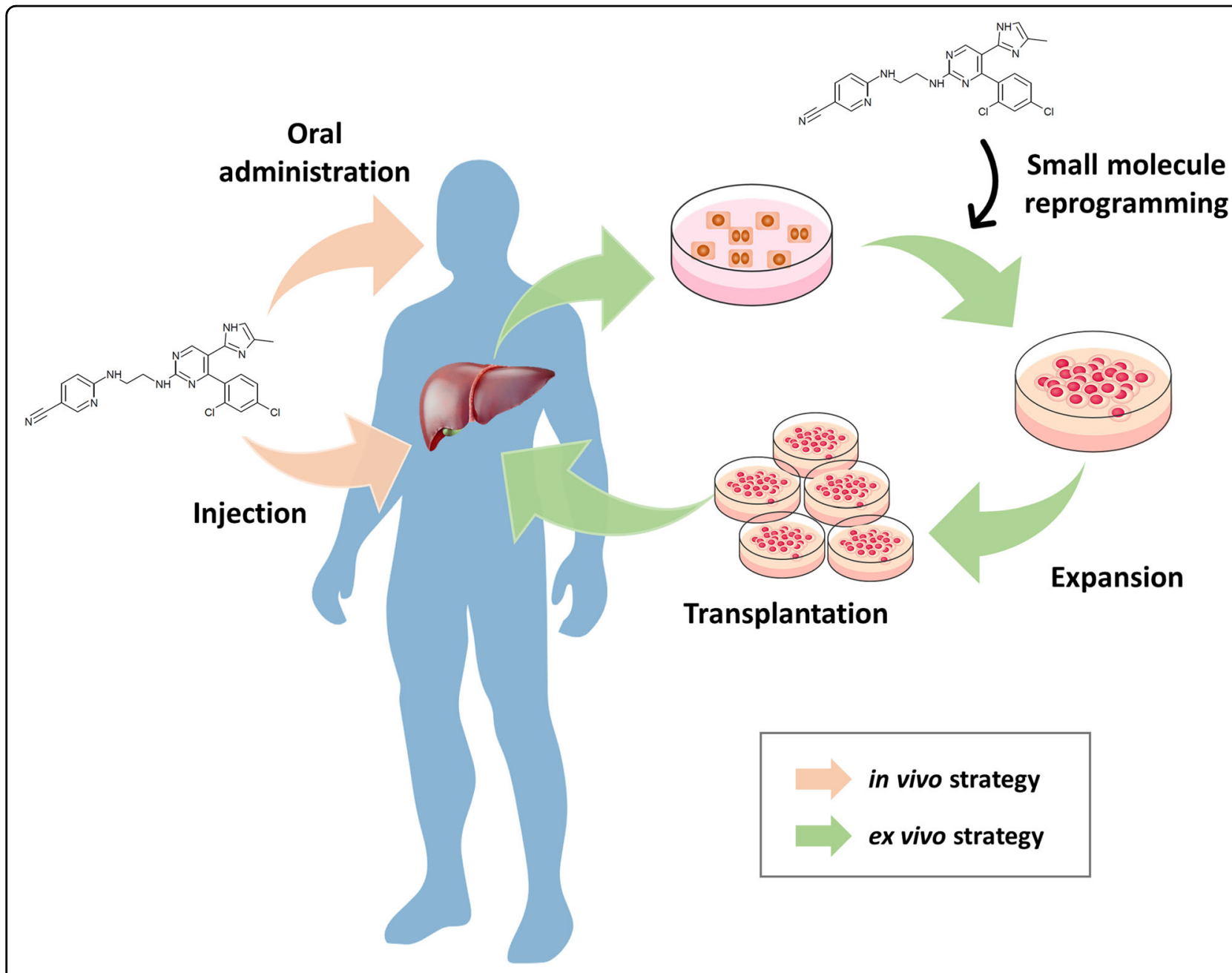

Fig. 5 Future challenges for small-molecule-mediated clinical trials. The future direction of personalized medicine is through small molecules. An ex vivo strategy can generate the desired cells through in vitro reprogramming using small molecules, and these cells are then expanded and transplanted into patients (green arrow). The in vivo strategy is to apply a small-molecule combination directly to patients via oral administration or injection (pink arrow).

administration, field injection, and intravenous injection. The next steps toward clinical application will involve confirming the possibility of inducing tumorigenicity and determining the proper concentration, combination of small molecules, and treatment times in vivo.

\section{Conclusion}

Cellular reprogramming provides the possibility of clinical application of stem cells for drug screening, disease modeling, artificial organ development, and cell therapy. Recently, to address the issue of ectopic gene expression and virus incorporation into chromosomes, reprogramming using small molecules was introduced. However, as with other stem cells, the functional maturation of reprogrammed cells and the efficiency of their reprogramming need to be increased, and a more complete understanding of the reprogramming mechanisms is required. However, the development of small-molecule-mediated reprogramming is eventually expected to be used for cell therapy, drug screening, personalized medicine, disease modeling, and even understanding regenerative medicine.

\section{Acknowledgements}

This study was carried out with the support of the "Cooperative Research Program for Agriculture Science and Technology Development (Project No. PJ01100202)" Rural Development Administration, Republic of Korea.

Conflict of interest

The authors declare that they have no conflict of interest.

\section{Publisher's note}

Springer Nature remains neutral with regard to jurisdictional claims in published maps and institutional affiliations. 
Received: 5 August 2019 Revised: 1 December 2019 Accepted: 27 December 2019.

Published online: 20 February 2020

\section{References}

1. Takahashi, K. \& Yamanaka, S. Induction of pluripotent stem cells from mouse embryonic and adult fibroblast cultures by defined factors. Cell 126, 663-676 (2006).

2. Takahashi, K. et al. Induction of pluripotent stem cells from adult human fibroblasts by defined factors. Cell 131, 861-872 (2007).

3. $Y u, J$. et al. Induced pluripotent stem cell lines derived from human somatic cells. Science 318, 1917-1920 (2007).

4. Dimos, J. T. et al. Induced pluripotent stem cells generated from patients with ALS can be differentiated into motor neurons. Science 321, 1218-1221 (2008).

5. Chambers, S. M. et al. Highly efficient neural conversion of human ES and iPS cells by dual inhibition of SMAD signaling. Nat. Biotechnol. 27, 275-280 (2009).

6. Karumbayaram, S. et al. Directed differentiation of human-induced pluripotent stem cells generates active motor neurons. Stem Cells 27, 806-811 (2009).

7. Hirami, Y. et al. Generation of retinal cells from mouse and human induced pluripotent stem cells. Neurosci. Lett. 458, 126-131 (2009).

8. Tateishi, K et al. Generation of insulin-secreting islet-like clusters from human skin fibroblasts. J. Biol. Chem. 283, 31601-31607 (2008)

9. Zhang, D. et al. Highly efficient differentiation of human ES cells and iPS cells into mature pancreatic insulin-producing cells. Cell Res. 19, 429-438 (2009).

10. Karner, E. et al. Differentiation of human embryonic stem cells into osteogenic or hematopoietic lineages: a dose-dependent effect of osterix over-expression. J. Cell. Physiol. 218, 323-333 (2009).

11. Choi, K. D. et al. Hematopoietic and endothelial differentiation of human induced pluripotent stem cells. Stem Cells 27, 559-567 (2009).

12. Zhang, J. et al. Functional cardiomyocytes derived from human induced pluripotent stem cells. Circulation Res. 104, e30-e41 (2009).

13. Gai, H. et al. Generation and characterization of functional cardiomyocytes using induced pluripotent stem cells derived from human fibroblasts. Cell Biol. Int. 33, 1184-1193 (2009).

14. Taura, D. et al. Adipogenic differentiation of human induced pluripotent stem cells: comparison with that of human embryonic stem cells. FEBS Lett. $\mathbf{5 8 3}$ 1029-1033 (2009).

15. Taura, D. et al. Induction and isolation of vascular cells from human induced pluripotent stem cells-brief report. Arteriosclerosis Thrombosis Vasc. Biol. 29 1100-1103 (2009).

16. Song, Z. et al. Efficient generation of hepatocyte-like cells from human induced pluripotent stem cells. Cell Res. 19, 1233-1242 (2009).

17. Si-Tayeb, K. et al. Highly efficient generation of human hepatocyte-like cells from induced pluripotent stem cells. Hepatology 51, 297-305 (2010).

18. Graf, T. \& Enver, T. Forcing cells to change lineages. Nature 462, 587-594 (2009).

19. Vierbuchen, T. et al. Direct conversion of fibroblasts to functional neurons by defined factors. Nature 463, 1035-1041 (2010).

20. leda, M. et al. Direct reprogramming of fibroblasts into functional cardiomyocytes by defined factors. Cell 142, 375-386 (2010).

21. Huang, P. et al. Induction of functional hepatocyte-like cells from mouse fibroblasts by defined factors. Nature 475, 386-389 (2011).

22. Gonzalez, F., Boue, S. \& Izpisua Belmonte, J. C. Methods for making induced pluripotent stem cells: reprogramming a la carte. Nat. Rev. Genet. 12, 231-242 (2011).

23. $\mathrm{Xu}, \mathrm{J} ., \mathrm{Du}, \mathrm{Y}$. \& Deng, $\mathrm{H}$. Direct lineage reprogramming: strategies, mechanisms, and applications. Cell Stem Cell 16, 119-134 (2015).

24. Hou, P. et al. Pluripotent stem cells induced from mouse somatic cells by small-molecule compounds. Science 341, 651-654 (2013).

25. $Y \mathrm{e}_{\text {, J. }}$. et al. Pluripotent stem cells induced from mouse neural stem cells and small intestinal epithelial cells by small molecule compounds. Cell Res. 26, 34-45 (2016)

26. Ying, Q. L. et al. The ground state of embryonic stem cell self-renewal. Nature 453, 519-523 (2008)

27. Lindvall, O., Kokaia, Z. \& Martinez-Serrano, A. Stem cell therapy for human neurodegenerative disorders-how to make it work. Nat. Med. 10(Suppl), S42-S50 (2004)

28. Cheng, L. et al. Generation of neural progenitor cells by chemical cocktails and hypoxia. Cell Res. 24, 665-679 (2014).
29. Huangfu, D. et al. Induction of pluripotent stem cells by defined factors is greatly improved by small-molecule compounds. Nat. Biotechnol. 26, 795-797 (2008).

30. Ichida, J. K. et al. A small-molecule inhibitor of tgf-Beta signaling replaces sox2 in reprogramming by inducing nanog. Cell Stem Cell 5, 491-503 (2009).

31. Yoshida, Y., Takahashi, K., Okita, K., Ichisaka, T. \& Yamanaka, S. Hypoxia enhances the generation of induced pluripotent stem cells. Cell Stem Cell 5, 237-241 (2009).

32. Zhang, M. et al. Pharmacological reprogramming of fibroblasts into neural stem cells by signaling-directed transcriptional activation. Cell Stem Cell $\mathbf{1 8}$ 653-667 (2016).

33. Gritti, A. et al. Multipotential stem cells from the adult mouse brain proliferate and self-renew in response to basic fibroblast growth factor. J. Neurosci. Off. J. Soc. Neurosci. 16, 1091-1100 (1996).

34. Li, W. et al. Rapid induction and long-term self-renewal of primitive neural precursors from human embryonic stem cells by small molecule inhibitors. Proc. Natl Acad. Sci. USA 108, 8299-8304 (2011).

35. Smith, J. R. et al. Inhibition of Activin/Nodal signaling promotes specification of human embryonic stem cells into neuroectoderm. Dev. Biol. 313, 107-117 (2008).

36. Hu, W. et al. Direct conversion of normal and Alzheimer's disease human fibroblasts into neuronal cells by small molecules. Cell Stem Cell 17, 204-212 (2015).

37. Zhang, L. et al. Small molecules efficiently reprogram human astroglial cells into functional neurons. Cell Stem Cell 17, 735-747 (2015).

38. Guo, Z. et al. In vivo direct reprogramming of reactive glial cells into functional neurons after brain injury and in an Alzheimer's disease model. Cell Stem Cell 14, 188-202 (2014)

39. Rodriguez-Martinez, G. \& Velasco, I. Activin and TGF-beta effects on brain development and neural stem cells. CNS Neurol. Disord. Drug Targets 11 844-855 (2012).

40. Gross, R. E. et al. Bone morphogenetic proteins promote astroglial lineage commitment by mammalian subventricular zone progenitor cells. Neuron 17 , 595-606 (1996)

41. Maden, M. Retinoid signalling in the development of the central nervous system. Nat. Rev. Neurosci. 3, 843-853 (2002).

42. Lin, T. et al. A chemical platform for improved induction of human iPSCs. Nat Methods 6, 805-808 (2009).

43. Watanabe, K. et al. A ROCK inhibitor permits survival of dissociated human embryonic stem cells. Nat. Biotechnol. 25, 681-686 (2007).

44. Hur, E. M. \& Zhou, F. Q. GSK3 signalling in neural development. Nat. Rev Neurosci. 11, 539-551 (2010).

45. Li, S. et al. GSK3 temporally regulates neurogenin 2 proneural activity in the neocortex. J. Neurosci. Off. J. Soc. Neurosci. 32, 7791-7805 (2012).

46. Borghese, L. et al. Inhibition of notch signaling in human embryonic stem cellderived neural stem cells delays G1/S phase transition and accelerates neuronal differentiation in vitro and in vivo. Stem Cells 28, 955-964 (2010).

47. Qu, Q. et al. High-efficiency motor neuron differentiation from human pluripotent stem cells and the function of Islet-1. Nat. Commun. 5, 3449 (2014).

48. Lie, D. C. et al. Wnt signalling regulates adult hippocampal neurogenesis Nature 437, 1370-1375 (2005).

49. Qian, L. et al. In vivo reprogramming of murine cardiac fibroblasts into induced cardiomyocytes. Nature 485, 593-598 (2012).

50. Song, $\mathrm{K}$. et al. Heart repair by reprogramming non-myocytes with cardiac transcription factors. Nature 485, 599-604 (2012).

51. Jayawardena, T. M. et al. MicroRNA-mediated in vitro and in vivo direct reprogramming of cardiac fibroblasts to cardiomyocytes. Circulation Res. 110 1465-1473 (2012).

52. Efe, J. A. et al. Conversion of mouse fibroblasts into cardiomyocytes using a direct reprogramming strategy. Nat. Cell Biol. 13, 215-222 (2011).

53. Wang, $H$. et al. Small molecules enable cardiac reprogramming of mouse fibroblasts with a single factor, Oct4. Cell Rep. 6, 951-960 (2014).

54. Fu, Y. et al. Direct reprogramming of mouse fibroblasts into cardiomyocytes with chemical cocktails. Cell Res. 25, 1013-1024 (2015).

55. Kong, Y. P., Carrion, B., Singh, R. K. \& Putnam, A. J. Matrix identity and tractional forces influence indirect cardiac reprogramming. Sci. Rep. 3, 3474 (2013).

56. Mureli, S. et al. Mesenchymal stem cells improve cardiac conduction by upregulation of connexin 43 through paracrine signaling. Am. J. Physiol. Heart Circulatory Physiol. 304, H600-H609 (2013) 
57. Zouein, F. A., Kurdi, M. \& Booz, G. W. LIF and the heart: just another brick in the wall? Eur. Cytokine Netw. 24, 11-19 (2013).

58. Frias, M. A. \& Montessuit, C. JAK-STAT signaling and myocardial glucose metabolism. Jak. Stat. 2, e26458 (2013).

59. Cao, N. et al. Conversion of human fibroblasts into functional cardiomyocytes by small molecules. Science 352, 1216-1220 (2016).

60. Burridge, P. W., Keller, G., Gold, J. D. \& Wu, J. C. Production of de novo cardiomyocytes: human pluripotent stem cell differentiation and direct reprogramming. Cell Stem Cell 10, 16-28 (2012).

61. Srivastava, D. \& Yu, P. Recent advances in direct cardiac reprogramming. Curr. Opin. Genet. Dev. 34, 77-81 (2015).

62. Le Bras, A. Basic research: Epigenetic map of heart development and disease. Nat. Rev. Cardiol. 15, 197 (2018).

63. Bae, S. H. Clinical application of stem cells in liver diseases. Korean J. Hepatol. 14, 309-317 (2008).

64. Kwon, Y. J., Lee, K. G. \& Choi, D. Clinical implications of advances in liver regeneration. Clin. Mol. Hepatol. 21, 7-13 (2015).

65. Bae, S. H. [Problems and prospects of stem cells for clinical use in hepatology]. Korean J. Hepatol. 11, 106-115 (2005).

66. Miyajima, A., Tanaka, M. \& Itoh, T. Stem/progenitor cells in liver development, homeostasis, regeneration, and reprogramming. Cell Stem Cell 14, 561-574 (2014).

67. Lu, W. Y. et al. Hepatic progenitor cells of biliary origin with liver repopulation capacity. Nat. Cell Biol. 17, 971-983 (2015).

68. Tarlow, B. D. et al. Bipotential adult liver progenitors are derived from chronically injured mature hepatocytes. Cell Stem Cell 15, 605-618 (2014).

69. Yimlamai, D. et al. Hippo pathway activity influences liver cell fate. Cell 157, 1324-1338 (2014).

70. Katsuda, T. et al. Conversion of terminally committed hepatocytes to culturable bipotent progenitor cells with regenerative capacity. Cell Stem Cell 20, 41-55 (2017).

71. Tsutsui, H. et al. An optimized small molecule inhibitor cocktail supports longterm maintenance of human embryonic stem cells. Nat. Commun. 2, 167 (2011).

72. Kawamata, M. \& Ochiya, T. Generation of genetically modified rats from embryonic stem cells. Proc. Natl Acad. Sci. USA 107, 14223-14228 (2010).

73. Chen, Q., Kon, J., Ooe, H., Sasaki, K. \& Mitaka, T. Selective proliferation of rat hepatocyte progenitor cells in serum-free culture. Nat. Protoc. 2, 1197-1205 (2007).

74. Kamiya, A., Kojima, N., Kinoshita, T., Sakai, Y. \& Miyaijma, A. Maturation of fetal hepatocytes in vitro by extracellular matrices and oncostatin $\mathrm{M}$ : induction of tryptophan oxygenase. Hepatology 35, 1351-1359 (2002).

75. Tateno, C. et al. Generation of novel chimeric mice with humanized livers by using hemizygous CDNA-uPASSCID mice. PloS ONE 10, e0142145 (2015).

76. Kim, Y. et al. Small molecule-mediated reprogramming of human hepatocytes into bipotent progenitor cells. J. Hepatol. 70, 97-107 (2019).

77. Kitade, M. et al. Specific fate decisions in adult hepatic progenitor cells driven by MET and EGFR signaling. Genes Dev. 27, 1706-1717 (2013).

78. Yin, L., Lynch, D., llic, Z. \& Sell, S. Proliferation and differentiation of ductular progenitor cells and littoral cells during the regeneration of the rat liver to CCI4/2-AAF injury. Histol. Histopathol. 17, 65-81 (2002).
79. Fu, G. B. et al. Expansion and differentiation of human hepatocyte-derived liver progenitor-like cells and their use for the study of hepatotropic pathogens. Cell Res. 29, 8-22 (2019).

80. Zhang, K. et al. In vitro expansion of primary human hepatocytes with efficient liver repopulation capacity. cell. stem cell. 23, 806-819. e804 (2018).

81. Johnson, M., Koukoulis, G., Matsumoto, K, Nakamura, T. \& Iyer, A. Hepatocyte growth factor induces proliferation and morphogenesis in nonparenchymal epithelial liver cells. Hepatology 17, 1052-1061 (1993).

82. Perugorria, M. J. et al. Wnt-beta-catenin signalling in liver development, health and disease. Nat. Rev. Gastroenterol. Hepatol. 16, 121-136 (2019).

83. Liu, X. et al. Brown adipose tissue transplantation improves whole-body energy metabolism. Cell Res. 23, 851-854 (2013).

84. Min, S. Y. et al. Human 'brite/beige' adipocytes develop from capillary networks, and their implantation improves metabolic homeostasis in mice. Nat. Med. 22, 312-318 (2016).

85. Cannon, B. \& Nedergaard, J. Brown adipose tissue: function and physiological significance. Physiol. Rev. 84, 277-359 (2004).

86. Seale, P. et al. PRDM16 controls a brown fat/skeletal muscle switch. Nature 454, 961-967 (2008).

87. Nie, B. et al. Brown adipogenic reprogramming induced by a small molecule. Cell Rep. 18, 624-635 (2017).

88. Alvarez, R. et al. Both retinoic-acid-receptor- and retinoid-X-receptordependent signalling pathways mediate the induction of the brown-adipose-tissue-uncoupling-protein-1 gene by retinoids. Biochem. J. 345(Pt 1), 91-97 (2000).

89. Lammert, E., Cleaver, O. \& Melton, D. Role of endothelial cells in early pancreas and liver development. Mechanisms Dev. 120, 59-64 (2003).

90. Liu, J. et al. Direct differentiation of hepatic stem-like WB cells into insulinproducing cells using small molecules. Sci. Rep. 3, 1185 (2013).

91. Clevers, H. \& Nusse, R. Wnt/beta-catenin signaling and disease. Cell $\mathbf{1 4 9}$ 1192-1205 (2012)

92. Li, R. et al. A mesenchymal-to-epithelial transition initiates and is required for the nuclear reprogramming of mouse fibroblasts. Cell Stem Cell 7, 51-63 (2010).

93. Wang, W. et al. Rapid and efficient reprogramming of somatic cells to induced pluripotent stem cells by retinoic acid receptor gamma and liver receptor homolog 1. Proc. Natl Acad. Sci. USA 108, 18283-18288 (2011).

94. Wobus, A. M. et al. Retinoic acid accelerates embryonic stem cell-derived cardiac differentiation and enhances development of ventricular cardiomyocytes. J. Mol. Cell. Cardiol. 29, 1525-1539 (1997).

95. Micallef, S. J. et al. Retinoic acid induces Pdx1-positive endoderm in differentiating mouse embryonic stem cells. Diabetes 54, 301-305 (2005).

96. Hochedlinger, K. \& Plath, K. Epigenetic reprogramming and induced pluripotency. Development 136, 509-523 (2009).

97. Pasque, V., Jullien, J., Miyamoto, K., Halley-Stott, R. P. \& Gurdon, J. B. Epigenetic factors influencing resistance to nuclear reprogramming. Trends Genet. 27, 516-525 (2011)

98. Li, Y. et al. Generation of iPSCs from mouse fibroblasts with a single gene, Oct4, and small molecules. Cell Res. 21, 196-204 (2011).

99. Park, J. T. et al. A crucial role of ROCK for alleviation of senescence-associated phenotype. Exp. Gerontol. 106, 8-15 (2018). 